\title{
Emergency department (ED) staff satisfaction analysis in response to COVID-19 crisis. Did the pandemic bring change in clinical practice and behaviours?
}

\author{
Muhammad Nauman Qureshi ${ }^{1 *}$ and Ferdinand Anguila Comia ${ }^{2}$ \\ ${ }^{1}$ Consultant Emergency department and Research director, King Faisal Specialist hospital and research centre, Riyadh, KSA \\ ${ }^{2}$ Registered nurse, Emergency medical services, King Faisal Specialist hospital and research centre, Riyadh, KSA
}

\begin{abstract}
COVID-19 crisis changed the spectrum of the Emergency department (ED) management and triggered a lot of challenges within the workforce. The staff whilst treating patients had to stay extra vigilant about cross infection. There was a set of rigorous processes put in place to meet the challenge of providing safe care to these highly infectious patients. These processes impacted ED staff, who were expected to provide a high-quality care amidst an extremely testing environment. We measured staff satisfaction across various domains, using a validated questionnaire. $150 \mathrm{ED}$ staff with different roles and skill sets were asked to score their satisfaction on the five point Likert scale ranging from1-5 ("Not satisfied" to "Most satisfied" in that order). 99\% of the recorded score was within the satisfaction domain and $87 \%$ staff claimed, the COVID-19 crisis changed their clinical practice and behaviour.
\end{abstract}

\section{Introduction}

COVID-19 pandemic overhauled emergency processes worldwide and King Faisal Specialist Hospital \& Research centre (KFSH), Riyadh was no exception. This 1600 bedded cancer and transplant facility with approximately $150 \mathrm{ED}$ staff caters for around 65000 patients annually. Its staff are geared up to deal with a vulnerable group of immunosuppressed patients, highly susceptible to infection. The health risks to the front end care providers greatly increased during the pandemic with a potential risk of cross infection to colleagues and family members. Our ED incorporated a number of internationally recommended safety measures for patients and staff, which completely transformed the ED environment and tested staff skills, commitment and resilience at length.

The changed working environment, processes \& constant use of personnel protective equipment (PPE) had an affect on the physical \& mental well-being of the care providers.

We wanted to measure the ED staff satisfaction around safety, new workplace processes \& support (offered to them by the department), during the COVID-19 crisis. We also wanted to know whether these processes brought any change in their clinical practice and behaviours.

\section{Measures in response to COVID-19 [1]}

There was screening at all ED entrances, where two care assistants measured temperature, offered hand sanitisers and face masks. ED registration desks had glass shields erected. A front end ED COVID-19 screening area was established, where a suspected infectious case was fast tracked to a designated negative pressure room. The ED waiting room was provided with a segregated respiratory waiting area, with a nurse taking care of that patient bubble. The triage was upgraded with a validated clinical screening tool to spot suspected COVID-19 cases (who somehow slipped the screening net) and to streamline them to appropriate areas.
Four negative pressure doored cubicles, with dedicated nurses were geared up for sick COVID-19 patients. Endotracheal intubations and other invasive procedures were carried out in one of these allocated rooms. Staff had regular drills about performance of barrier nursing, delivery of aerosol treatments and transfer of COVID-19 patients. There were clear guidelines about wearing personal protection equipment (PPE), which were regularly audited. Staff had to adopt social distancing protocols and were also subjected to regular COVID-19 PCR testing. Staff were expected to stay acquainted with the local and national COVID-19 updates. Staff involved in caring a suspected patient were subjected to rapid COVID-19 screening and quarantined as per local policy. A dedicated self isolation residential facility was provided for COVID-19 positive expatriate staff.

\section{Methodology}

We conducted a staff survey using a validated questionnaire (Figure 1) to measure satisfaction across various domains. They were asked to score their satisfaction on the five point Likert scale ranging from 1-5 (1 meant "Not satisfied" and 5 meant "Most satisfied"). A total of $150 \mathrm{ED}$ staff had the survey forms, hand delivered by the co-author. The surveyed staff included physicians, nurses (managers, bedside), paramedics, \& non-clinical staff (registration desk staff and ER coordinators). The forms after completion were deposited in a sealed box kept visible in a secured place.

*Correspondence to: Muhammad Nauman Qureshi, Consultant \& Research director, King Faisal Specialist Hospital \& Research centre; Assistant professor (adjunct), AlFaisal university, Riyadh, KSA, E-mail: qmuhammad@kfshrc.edu.sa

Received: November 30, 2020; Accepted: January 04, 2021; Published: January 07,2021 
We interpreted the results using staff numbers and percentages in each measured domain. The statistical analysis was done by using the software package SAS version 9.4 (SAS Institute Inc., Cary, NC, USA).

\section{Results}

1- Satisfaction with the screening facility: (Graph 1 \& Figure 1) Six (4\%) were "partly satisfied", 39(25\%) were satisfied, 65(44\%) were "more than satisfied", 40(27\%) were "most satisfied".

2- Satisfaction with the triage facilities: (Graph 2 \& Figure 2) Five (3\%) were partly Satisfied, 43 (29\%) were satisfied, 61(41\%) were "more than satisfied" and 40 (27\%) were "most satisfied".

3- Satisfaction regarding ambulance handovers: (Graph 3 \& Figure 3) Three (2\%) were "partly satisfied", 53 (34\%) were satisfied, 66 (45\%) were "more than satisfied", 28 (19\%) were "most satisfied".

4- Satisfaction with the provided PPE: (Graph 4 \& Figure 4) Six (4\%) were not satisfied, 8 (5\%) were "partly satisfied", 47 (34\%) were satisfied, 55 (35\%) were "more than satisfied", 34 (22\%) were "most satisfied".

5- Knowledge of the process for getting help in case of exposure:(Graph 5 \& Figure 5) One hundred \& forty six (97\%) said "yes" to this question.

6- Do you feel supported in the current crisis situation? (Graph 6 \& Figure 6) One hundred \& thirty nine (93\%) answered "yes" to this question.

7- Did the COVID-19 crisis change the clinical practice and behavior? (Graph 7 \& Figure 7) 87\% said "yes" to this question.

8- Overall satisfaction of the ED staff with the ED processes: (Graph 7 \& Figure 7) 46\% "most satisfied", 26\% "more than satisfied", 25\% satisfied, $4 \%$ "partly satisfied", $1 \%$ "not satisfied"

\section{Staff distribution (domain 1 )}

50

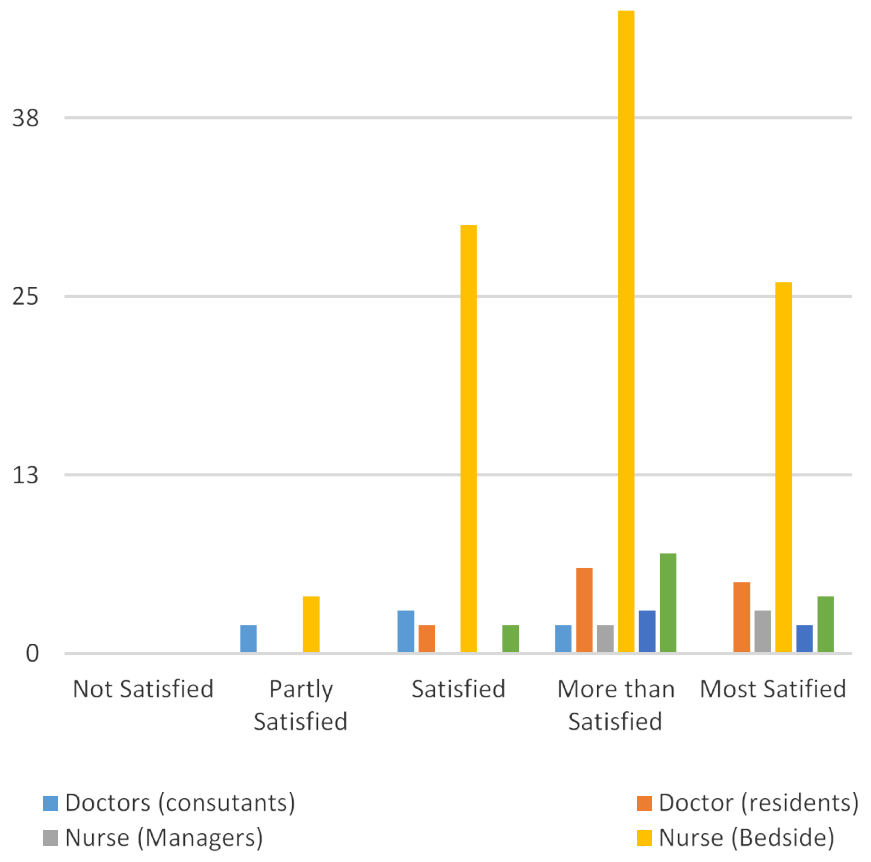

Figure 1. Staff satisfaction with the screening facilities
107 (71\%) surveyed staff were bedside nurses. In addition it included 20 (13\%) physicians, 5 (3\%) paramedics, 5 (3\%) nurse managers and 13 (9\%) non-clinical staff (Registration desk, ward clerk, coordinators).

"More than satisfied" response dominated the score and ranged from $35-45 \%$ across various domains. The second common response was "satisfied" which ranged from 25-34\%. "Most satisfied" ranged from $19-27 \%$ followed by "partly satisfied" which was from $2-5 \%$. "Not satisfied" was only answered by $4 \%$ staff in response to the PPE question and by $1 \%$ in response to the overall satisfaction with the ED processes question.

\section{Discussion}

Workforce's productivity and quality is highly dependent on their morale, which is directly proportional to satisfaction at work. ED staff at the front line, face the most stress, due to high risk of exposure. The highly infectious and deadly COVID-19 pandemic, multiplied their stress levels [2]. The ED management triggered additional processes based on international standards, to combat the crisis. The staff were the key stakeholders to implement these processes, hence their satisfaction was very important to achieve the desired outcome through these processes $[3,4]$.

Surveyed ED staff had more inclusion of bedside nurses which could be due to their higher number in the department. As most of the new process changes was around barrier nursing, their feedback reflects the overall staff satisfaction.

The upgrading of the existing policies and protocols led to complete transformation of key areas within ED.

Electronic gate pass was introduced to check the employee COVID-19 negative status on entry. Entrances were manned by

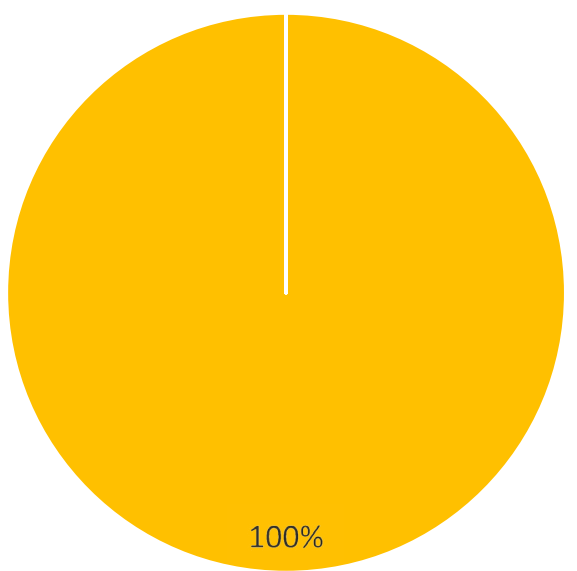

- How satisfied are you with the screening facilities?

Graph 1 


\section{Staff distribution (domain 2)}

40
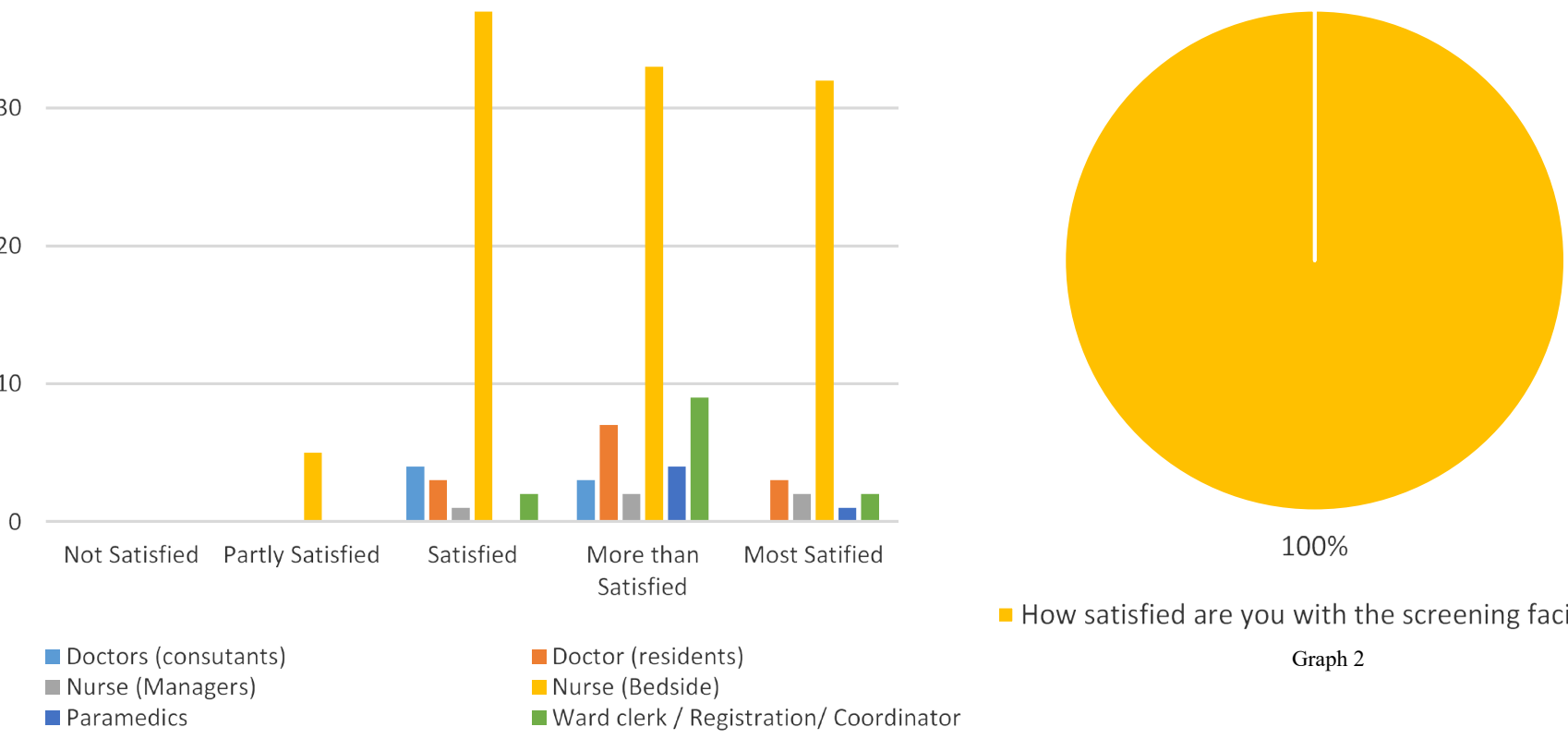

How satisfied are you with the screening facilities? Graph 2

Figure 2. Staff satisfaction with triage facilities

\section{Staff distribution (domain 3)}

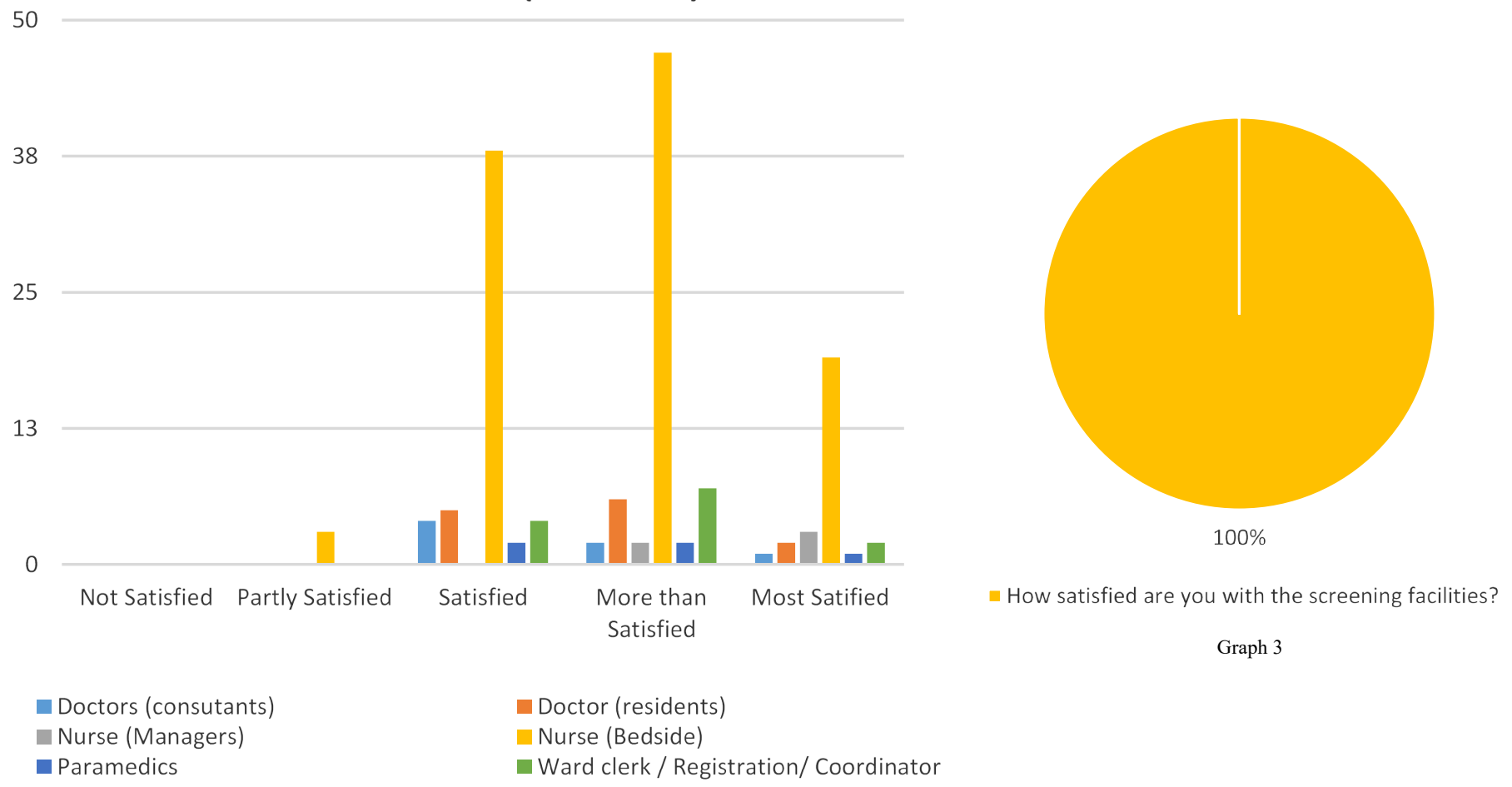

Figure 3. Staff satisfaction with handover of ambulance covid-19 patients 

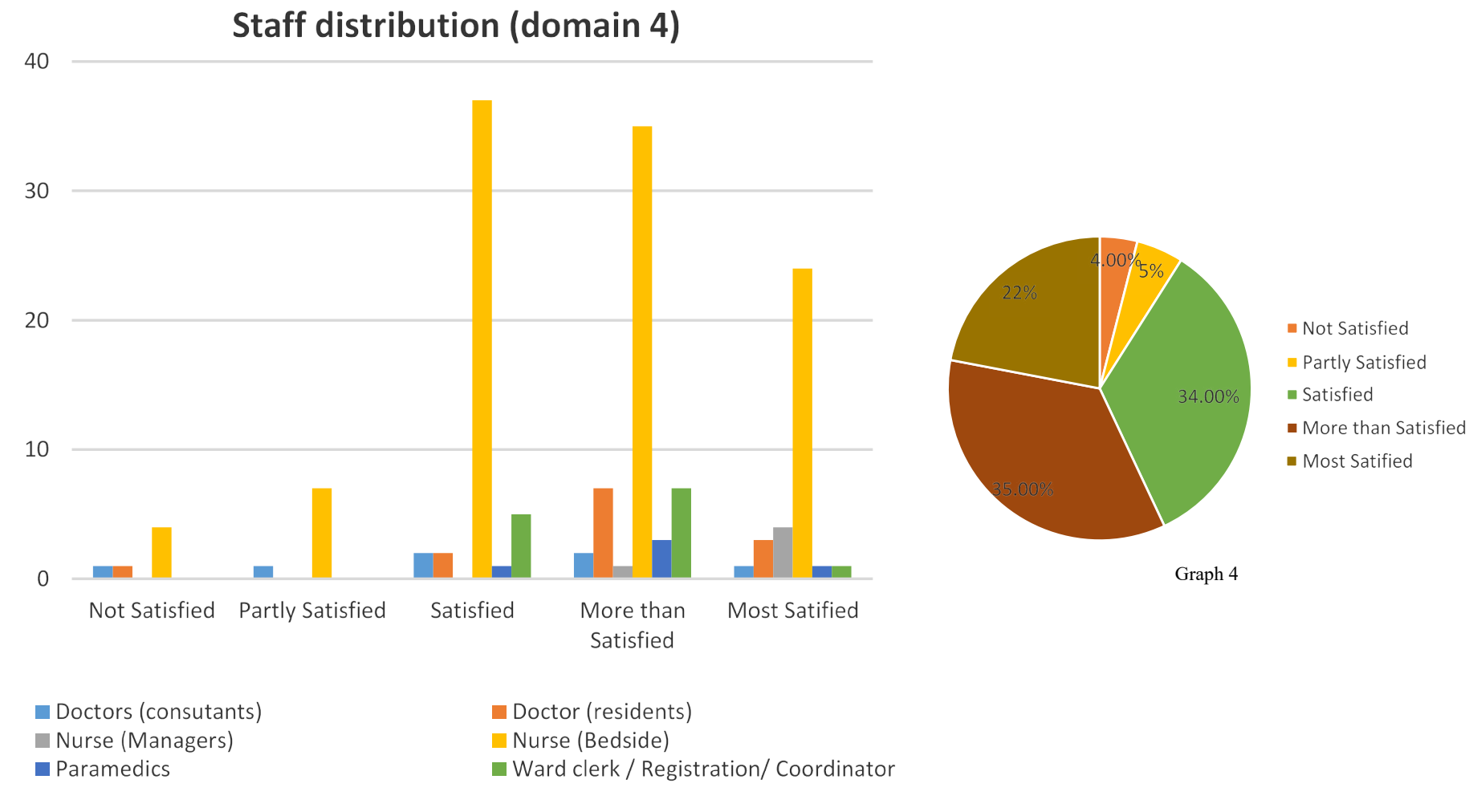

Figure 4. Staff satisfaction about the provided personal protective equipment

\section{Staff distribution (domain 5)}

120
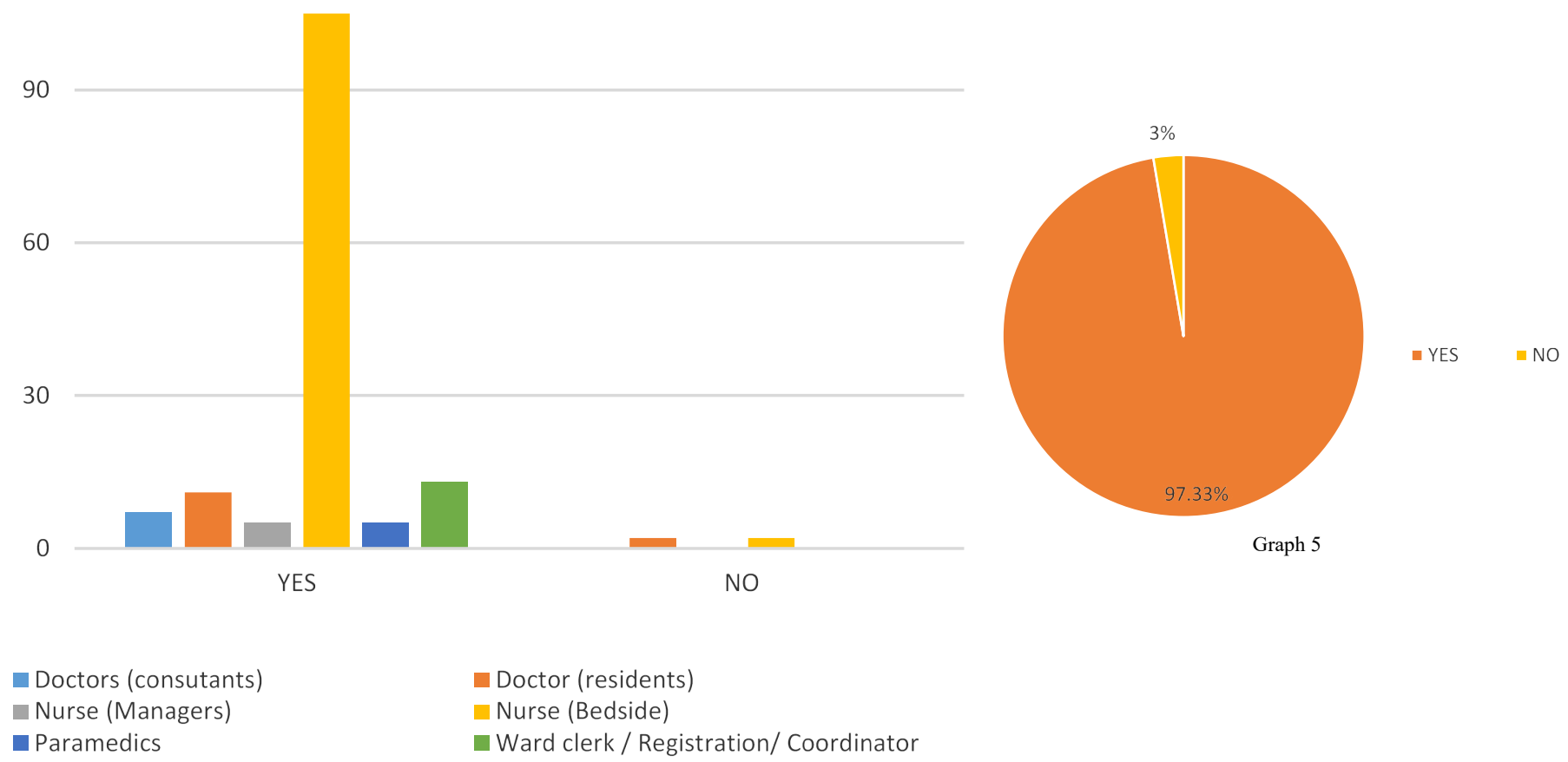

Figure 5. Staff awareness of getting support in case of exposure to covid-19 case 


\section{Staff distribution (domain 6)}

120
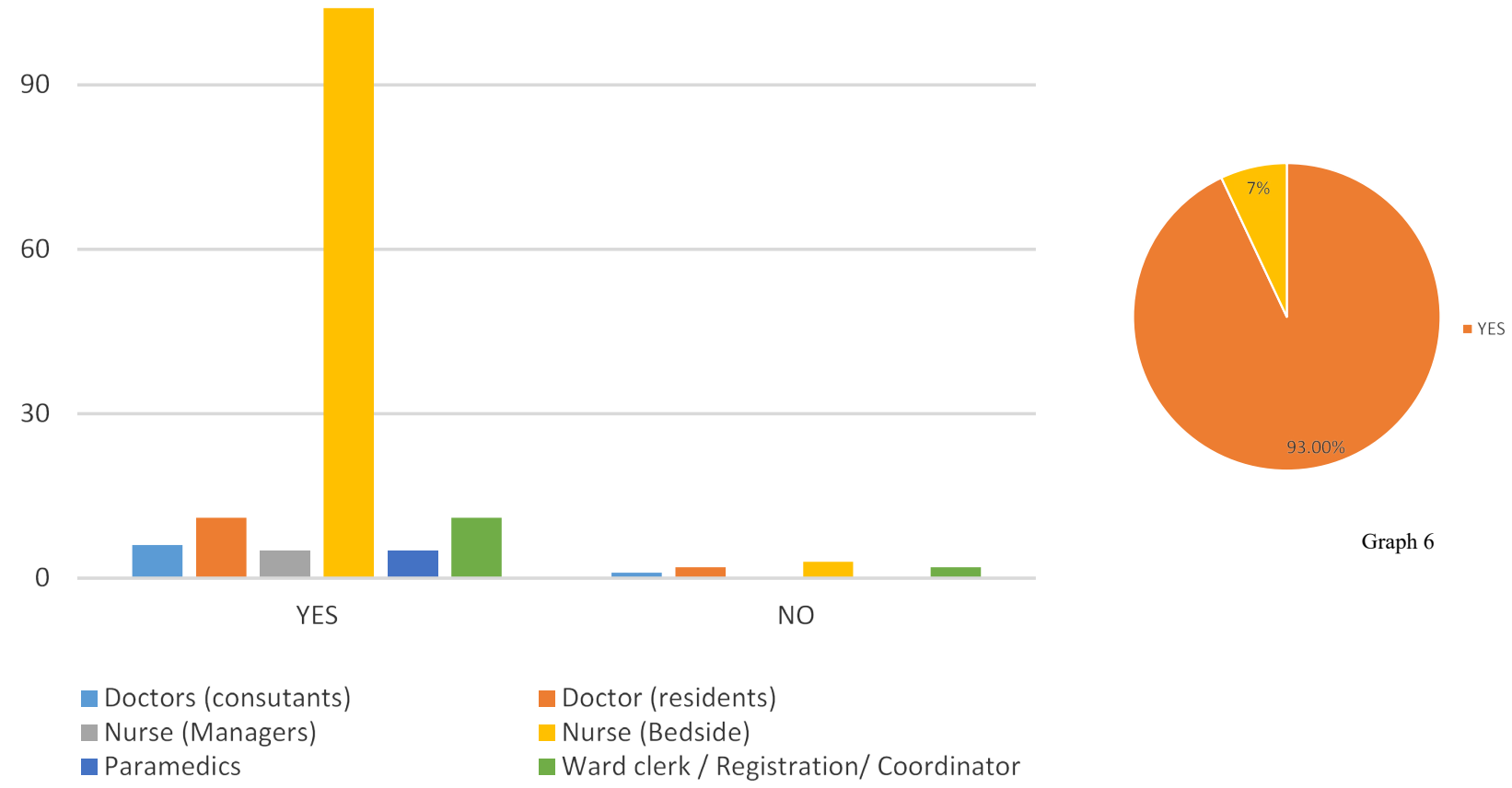

Graph 6

(Managers)

- Ward clerk / Registration/ Coordinator

Figure 6. Staff feeling of being supported during the covid-19 crisis situation

\section{Staff distribution (domain 7)}

100
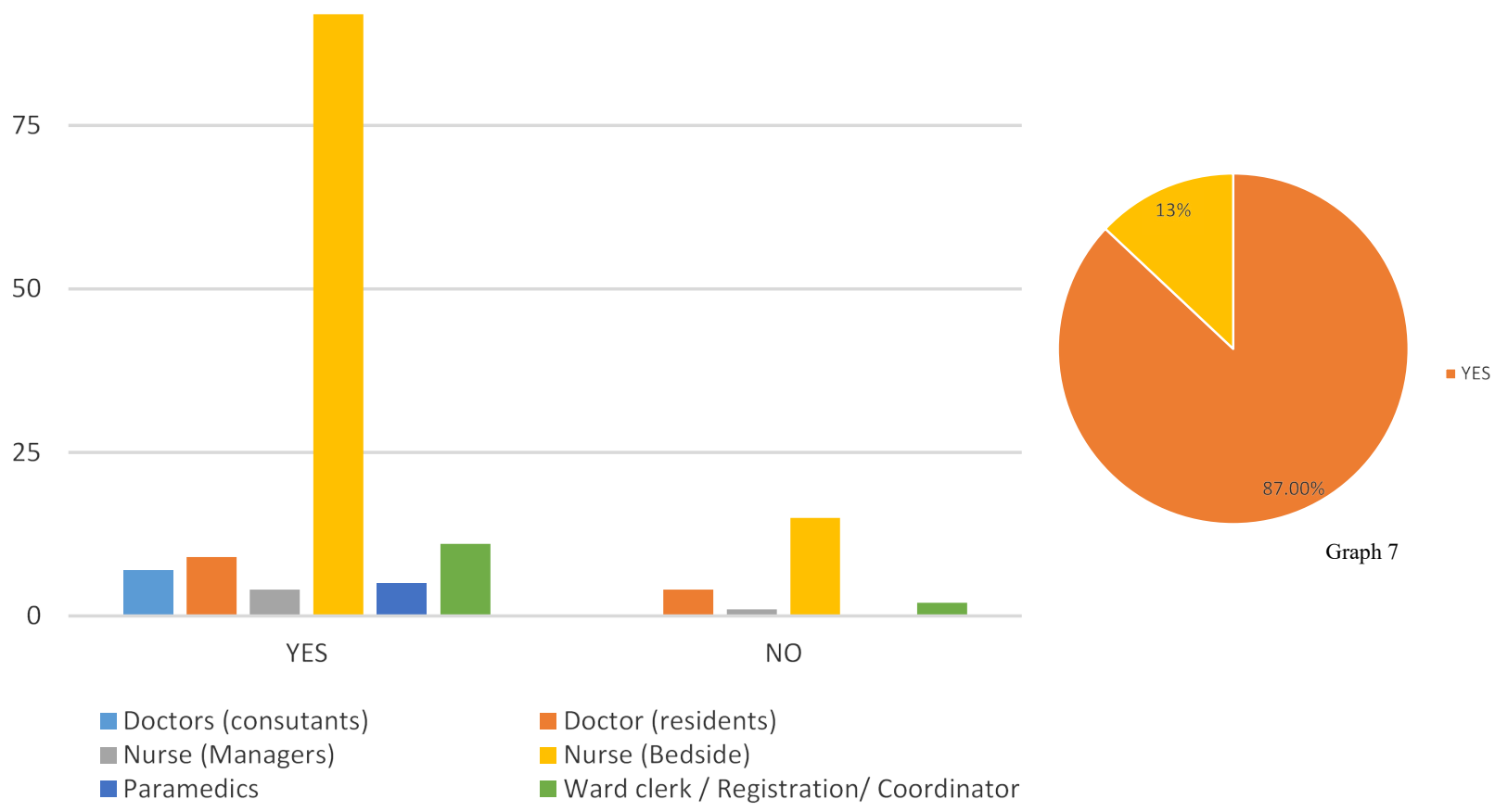

Figure 7. Do you feel any change in your clinical practice and behavior after dealing with the covid-19 crisis 


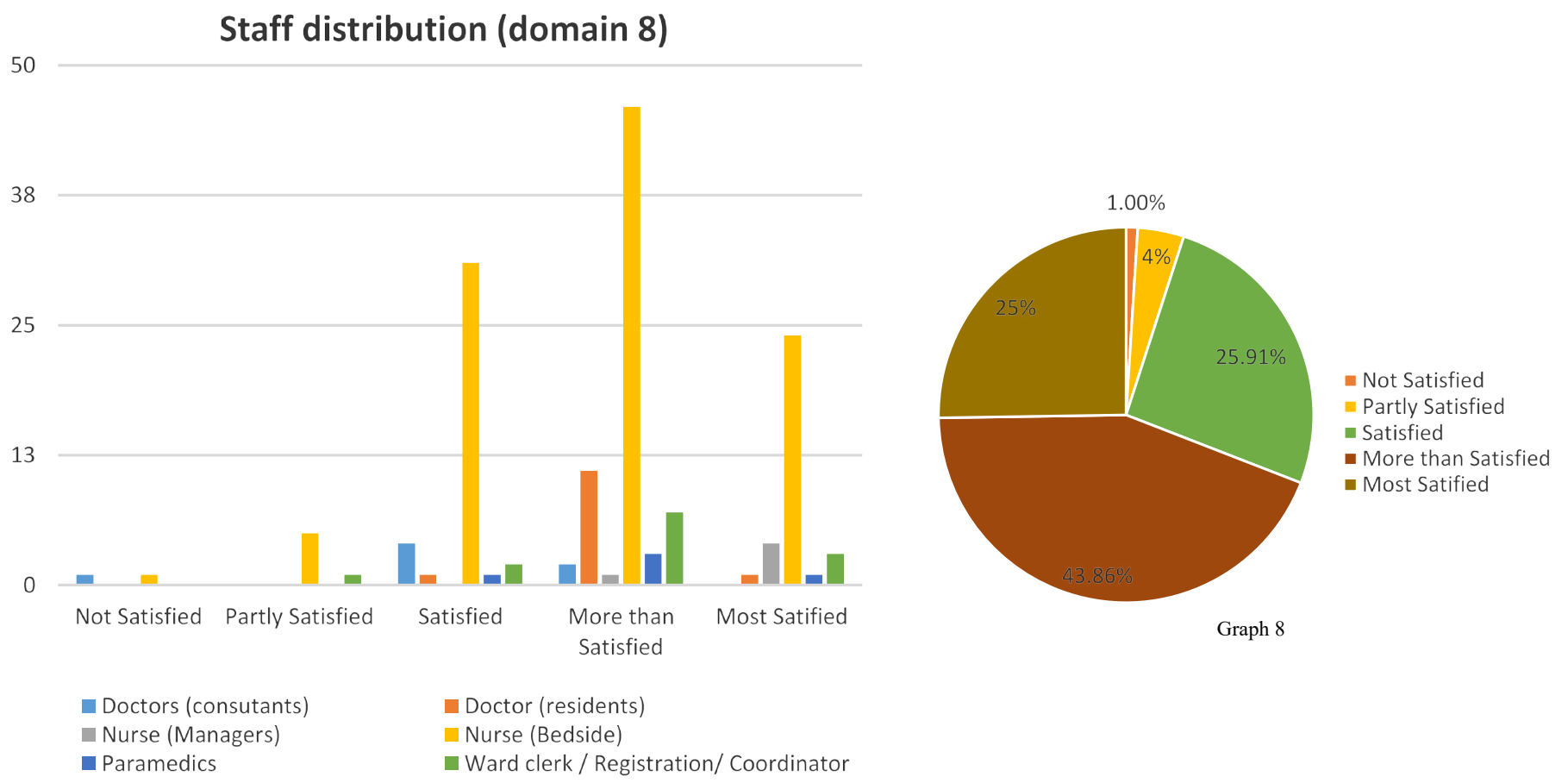

Figure 8. How satisfied are you with the processes adopted by your ed in relation to covid-19 crisis

nursing personnel, to check temperature, provide face masks hand sanitization [5].

The Ministry of Health's approved "severe acute respiratory screening tool" (SARI) for MERS-CoV (Middle Eastern Respiratory Syndrome) was upgraded for COVID-19 screening at "Triage" (Figure 9). COVID-19 suspected patients were shifted to the designated rooms from triage and ED clinician was immediately informed. $41 \%$ of the ED staff were "more than satisfied" with the Triage processes [6,7].

Ambulance patients and paramedics were screened separately on arrival from a designated ED entrance. Suspected patients with high SARI scores were held in the ambulance bay, equipped with hepafilters. Screening personnel at the entrance jointly carved a plan with the triage nurse for allocation of appropriate clinical space. Forty-five percent staff expressed "More than Satisfied" on this incorporated measure [8].

Personal Protective Equipment (PPE) had a temporary shortage during the pandemic surge, which led to rationing of N95 face masks. The supply catered for the needs of the ED staff but the visiting heath care providers from another other specialty were encouraged to bring their own masks from their clinical areas. The limited resource did affect staff satisfaction scores [9].

The EMS Nursing created a "tracing team" to focus on the cases with exposure to Covid-19. ED staff were also kept informed through huddles, meetings and emails. The Organization developed a COVID-19 central hotline with involvement of Infection control team. The flowcharts for managing the staff, exposed with COVID-19 were created. An electronic COVID-19 dashboard was designed in the KFSH portal to keep the employees updated $[10,11]$.

Ninety-seven percent of the staff expressed that they had knowledge of getting help in case of exposure to COVID-19. Clear communication through group emails kept the staff informed about support mechanisms [12].

Other ED support processes were introduced, which enhanced staff satisfaction.

ED staff working patterns were staggered to avoid exposure, whilst keeping a critical mass of the staff on the floor.

"Staff safety and well-being project" was initiated to help staff cope with stress. Counselling for ED staff was provided to support their mental health.

The over-all percentage of ED staff, who felt supported in the current crisis situation was ninety-three percent of the total sample size. Recognition award was introduced by the nursing team to reward exemplary compliance with the new measures, during the pandemic [13].

The quarterly patient experience survey of the KFSH patients in 2020, during the pandemic revealed highest satisfaction amongst the ED patients when compared to other areas ( $78.3 \%$ vs $75 \%)$. This was also higher than the preceding quarter.

The "zero harm scoreboard" which records any ED patient related clinical incidences maintained zero harm during the period from March to July 2020, which was the peak of pandemic in the country. Saudi Central Board for Accreditation of Healthcare Institutions visited KFSH in July 2020 and commended ED handling and management of COVID-19 cases [14].

The over-all percentage of ED staff who felt that there were changes in their clinical practice and behaviour as a result of COVID-19 crisis was eighty seven percent of the total sample size.

\section{Conclusions}

A significant proportion of KFSH ED staff expressed satisfaction towards the processes introduced in response to COVID-19 pandemic. 


\section{Limitations}

The surveyed ED staff are from a single large tertiary care centre in KSA. These satisfaction scores may be different from other ED staff scores, which in turn will be dependent on the processes, introduced in those departments.

\section{References}

1. Nauman QM, AlRajhi A (2020) Challenge of COVID-19 crisis managed by emergency department of a big tertiary centre in Saudi Arabia. International Journal of Pediatrics and Adolescent Medicine 7(3): 147-152. [Crossref]

2. Holly B, Bermingham F, Johnson G, Tabner A (2020) Mitigating the psychological impact of COVID-19 on healthcare workers: a digital learning package. International Journal of Environmental Research and Public Health 17(9): 2997. [Crossref]

3. Elise G, Porter JE, Morphet J (2017) An exploration of emergency nurses' perceptions, attitudes and experience of teamwork in the emergency department. Australasian Emergency Nursing Journal 20(2): 92-97. [Crossref]

4. Stacie H, Chen HC, Maughan D, Heaston S (2015) Factors that influence the development of compassion fatigue, burnout, and compassion satisfaction in emergency department nurses. Journal of Nursing Scholarship 47(2): 186-194. [Crossref]

5. Thomas HG, Olgers TG, Wortel RR, Noltes ME, Dercksen B, et al. (2018) The satisfaction regarding handovers between ambulance and emergency department nurses: an observational study. Scandinavian Journal of Trauma, Resuscitation and Emergency Medicine 26(1): 78. [Crossref]
6. Suárez M, Asenjo MBAM, Sánchez (2017) Job satisfaction among emergency department staff. Australasian Emergency Nursing Journal 20(1): 31-36. [Crossref]

7. Panagiota G, Mantas C, Dimitroula D, Mantis D, Hyphantis T (2010) General hospital staff worries, perceived sufficiency of information and associated psychological distress during the A/H1N1 influenza pandemic. BMC Infectious Diseases 10(1): 322. [Crossref]

8. Pandemic WHO (2009) update 109. WHO Global Alert and Response.

9. Rima S, Hawryluck L, Robinson S, Kasapinovic S, Fones C, et al. (2008) Impact on health care workers employed in high-risk areas during the Toronto SARS outbreak. Journal of Psychosomatic Research 64(2): 177-183. [Crossref]

10. Cindy WCT, Pang EPF, Lam LCW, Chiu HFK (2004) Severe acute respiratory syndrome (SARS) in Hong Kong in 2003: stress and psychological impact among frontline healthcare workers. Psychological Medicine 34(7): 1197. [Crossref]

11. YaMei B, Lin CC, Lin CY, Chen JY, Chue CM, et al. (2004) Survey of stress reactions among health care workers involved with the SARS outbreak. Psychiatric Services 55(9): 1055-1057. [Crossref]

12. Sarah D, Wilson S, Draper H, Gratus C, Greenfield S, et al. (2009) Will the NHS continue to function in an influenza pandemic? A survey of healthcare workers in the West Midlands, UK. BMC Public Health 9(1): 142. [Crossref]

13. Holly S, Leask J, Po K, MacIntyre CR (2009) "Will they just pack up and leave?"attitudes and intended behaviour of hospital health care workers during an influenza pandemic. BMC Health Services Research 9(1): 30. [Crossref]

14. Kati U, Kyngäs H (2009) Hospital nurses' job satisfaction: a literature review. Journal of Nursing Management 17(8): 1002-1010. [Crossref]

Copyright: $@ 2021$ Qureshi MN. This is an open-access article distributed under the terms of the Creative Commons Attribution License, which permits unrestricted use, distribution, and reproduction in any medium, provided the original author and source are credited. 